\title{
Historia metabólica de pacientes con antecedentes de diabetes gestacional. Seguimiento durante seis años (2013-2019)
}

\author{
Metabolic record of patients with history of gestational diabetes. \\ Follow-up of patients for six years (2013-2019)
}

\author{
Jorge L. Olivares ${ }^{1}$, Marisol G. Olivares ${ }^{2}$, Luciana Grilli ${ }^{3}$, Carina B. Verdasco ${ }^{4}$, Carolina Miglianelli ${ }^{5}$
}

\section{RESUMEN}

Introducción: en la provincia de La Pampa no hay registro del número de pacientes con diabetes gestacional (DG) que vuelve a control metabólico posparto, cuando se conoce el riesgo que evolucione a diabetes mellitus tipo 2 (DM2).

Objetivos: analizar en el Hospital Público Interzonal (Dr. Lucio Molas) el porcentaje de mujeres con DG que volvió a control metabólico posparto y desarrolló DM2 durante seis años de seguimiento.

Materiales y métodos: estudio descriptivo ambispectivo en 44 pacientes con DG diagnosticada en 2013 con seguimiento hasta el 31/12/2019. Se analizaron antecedentes previos de DG y familiares de diabetes (AFD), edad, semana de gestación del diagnóstico con glucemia/prueba de tolerancia oral a la glucosa (PTOG), índice de masa corporal (IMC), presión arterial, vía del parto, peso del recién nacido, HbA1c.

Resultados: en 2013 se efectuaron 1.238 partos, 44 pacientes $(3,7 \%)$ tenían DG diagnosticada, 68\% mediante PTOG. Presentaban $43 \%$ AFD, 38,6\% sobrepeso, 20,45\% obesidad, $68,2 \%$ cesáreas y $11,4 \%$ recién nacidos con alto peso. En seis años de seguimiento, $54,5 \%$ de estas pacientes realizó control metabólico; este grupo, a diferencia del grupo sin control, presentó mayor IMC al inicio del embarazo (t:2,103; $p<0,02)$, glucemia basal (t:10,59; $p<0,001)$, presión arterial sistólica (t:2,629; $p<0,01)$, diastólica (t:1,965; $p<0,05)$ y macrosomía fetal (4 vs 1). El 33,3\% manifestó DM2 predominantemente a partir de tres años del posparto.

Conclusiones: el $54,5 \%$ de las pacientes con DG concurrió a control metabólico posparto tardíamente, y el 33,3\% evolucionó a DM2; incluso estas pacientes presentaron mayor número de factores de riesgo obstétricos y cardiometabólicos que el grupo sin control al inicio del embarazo.

Palabras clave: diabetes gestacional; testeo posnatal para diabetes mellitus tipo 2; criterios diagnósticos; resultados maternos; resultados fetales.

Revista de la Sociedad Argentina de Diabetes 2021; Vol. 55 (13-20)

\section{ABSTRACT}

Introduction: there is no register of the number of women with gestational diabetes (GD) that have undergone metabolic post-partum control in the province of La Pampa, even though it is well-known the increased associated risk of becoming a diabetes type 2 patient (T2DM).

Objectives: to analyze at the Interzonal Public Hospital (Dr. Lucio Molas) the percentage of women with GD who returned to postpartum metabolic control and developed T2DM during six years of follow-up.

Materials and methods: descriptive ambispective study in 44 patients with GD diagnosed in 2013 with follow-up until 31 December 2019. Different characteristics were analyzed in all participants: previous history of GD and family members of diabetes (FHD), age, gestational week of the diagnosis with fasting glucose or oral glucose tolerance test (OGTT), body mass index (BMI), blood pressure, delivery route, newborn weight, glycated hemoglobin (HbA1C).

Results: a number of 1,238 of deliveries were performed in 2013, 44 women (3.7\%) developed GD diagnosed by OGTT (68\%), 43\% presented FHD, 38.6\% overweight, $20.45 \%$ obesity. Among the totality of deliveries $68.2 \%$ were by caesarea and $11.4 \%$ high birth weight newborns. In six years of follow-up, $54.5 \%$ of these patients underwent metabolic control, presenting this group, unlike the group without control, higher BMI at the beginning of pregnancy (t:2.103; $p<0.02)$, fasting blood glucose (t:10 .59; $p<0.001)$, systolic blood pressure (t:2.629; $p<0.01$ ), diastolic blood pressure ( $t ; 1.965 ; p<0.05)$ and fetal macrosomia (4 vs 1). 33.3\% developed T2DM predominantly from at three years postpartum.

Conclusions: the present study reported that $54.5 \%$ of women with GD performed a late metabolic postpartum control evolving 33.3\% developed T2DM presenting higher obstetric and cardiometabolic risk factors than the group without control at the beginning of pregnancy.

Key words: gestational diabetes; type 2 diabetes mellitus postnatal screening; diagnostic criteria; maternal outcomes; fetal outcomes.

Revista de la Sociedad Argentina de Diabetes 2021; Vol. 55 (13-20) 
1 Jefe del Departamento de Docencia e Investigación, Médico de Planta, Servicio de Endocrinología y Diabetes, Establecimiento Asistencial Dr. Lucio Molas, Profesor titular de la Universidad Nacional de la Pampa, Santa Rosa, La Pampa, Argentina

2 Residente de tercer año de Clínica Médica, Ministerio de Salud de la Nación, Sede de Residencias, Establecimiento Asistencial Dr. Lucio Molas, Santa Rosa, La Pampa, Argentina

3 Jefa del Servicio de Endocrinología y Diabetes, Establecimiento Asistencial Dr. Lucio Molas, Santa Rosa, La Pampa, Argentina

4 Lic. Obstétrica, Planta del Servicio de Tocoginecología, Establecimiento Asistencial Dr. Lucio Molas, Santa Rosa, La Pampa, Argentina
5 Médica de Planta, Servicio de Endocrinología y Diabetes, Establecimiento Asistencial Dr. Lucio Molas, Santa Rosa, La Pampa, Argentina

Contacto del autor: Jorge Luis Olivares

E-mail: jorgeluis57.olivares@gmail.com

Correspondencia: Emilio Mitre 340, CP6300, Santa Rosa,

La Pampa, Argentina

Fecha de trabajo recibido: 20/10/20

Fecha de trabajo aceptado: 17/12/20

Conflictos de interés: los autores declaran que no existe conflicto de interés

\section{INTRODUCCIÓN}

La diabetes gestacional (DG) se define como la alteración del metabolismo de los hidratos de carbono, de severidad variable, que se inicia o se reconoce por primera vez durante el embarazo. En cambio, la diabetes pregestacional es aquella que se manifiesta en pacientes ya diagnosticadas con diabetes mellitus tipo 1 ó 2 (DM1 o DM2) previo al embarazo'.

El tratamiento de la DG demanda el seguimiento con un equipo interdisciplinario constituido por obstetra/obstétrica, enfermera, nutricionista, endocrinólogo y otros integrantes del equipo de salud por el alto riesgo materno-fetal que presenta ${ }^{2}$. Durante el transcurso del embarazo, las pacientes con DG generalmente concurren a sus controles porque reconocen que la salud fetal puede comprometerse ${ }^{3}$. La falta de control adecuado implica un aumento de la morbimortalidad prenatal por diversas complicaciones asociadas como preeclamsia, eclampsia o descompensación metabólica severa² ${ }^{2}$. Debido a estas evidencias, el equipo de salud aconseja a toda puérpera con antecedente de DG repetir una prueba de tolerancia oral a la glucosa (PTOG) entre las 4 y 12 semanas posparto para evaluar si presenta DM2 $2^{1,4,5}$.

La DG tiene una prevalencia del 4,7 al $18 \%$ y se caracteriza por ser una patología con la posibilidad de que, al finalizar este estado fisiológico, la paciente normalice la función pancreática endógena, pero también que se reitere la alteración metabólica en próximos embarazos, por ello la importancia de la educación diabetológica, ${ }^{5,6}$. El antecedente de DG implica un aumento del riesgo de siete veces de desarrollar DM2 y de transformarse en un estado irreversible de la patología? Se ha determinado que el $50 \%$ de las pacientes que ha padecido DG puede manifestar DM2 entre 5 a 10 años posparto o ante un nuevo embarazo ${ }^{2}$. El grupo de estudio de la Asociación Internacional de Diabetes y Embarazo (IADPSG, sus siglas en inglés) determinó, en un seguimiento durante cinco años a 270 mujeres con antecedentes de DG, que el $26 \%$ evolucionó a DM2, mientras que en las embarazadas con tolerancia oral a la glucosa normal sólo evolucionó a DM2 el 4\% ${ }^{4}$. Kugishima et al. ${ }^{8}$ encontraron que el $10 \%$ de las embarazadas desarrolló DM2 en un rango entre seis y 292 semanas posparto y $11 \%$ reiteró DG en otro embarazo.

En un reciente estudio ${ }^{9}$ se estudiaron las complicaciones obstétricas ocurridas durante el embarazo en 280 pacientes que presentaban diabetes pregestacional (DM1 y DM2) o DG y se compararon con un grupo control de embarazadas. Las pacientes con DG que poseían antecedentes de partos pretérmino, multiparidad, neonatos grandes para edad gestacional, alto índice de masa corporal (IMC) pregestacional o excesiva ganancia de peso durante el embarazo presentaron mayor riesgo de tener recién nacidos con alto peso para la edad gestacional, con mayor incidencia de cesárea. Otros autores determinaron que en la DG debe considerarse como factor de riesgo la hipertrigliceridemia porque predispone al desarrollo de macrosomía fetal ${ }^{10}$. Se ha demostrado ${ }^{2,6}$ la importancia que tiene el seguimiento con plan alimentario de pacientes con DG que presentan sobrepeso/obesidad y antecedente de multiparidad, a partir de lo cual toman conciencia del manejo nutricional para su control metabólico en beneficio de la salud de su hijo.

Otra mirada de la importancia del seguimiento posparto y de la detección precoz de las alteraciones metabólicas en pacientes con antecedente de DG, es la potencialidad de prevenir, desde la Clínica Médica, la evolución a DM2 y evitar el desarrollo de complicaciones cardiovasculares ${ }^{11}$. 


\section{OBJETIVOS}

El interés por realizar esta investigación se basó en que, consultadas fuentes primarias del Servicio de Endocrinología y Diabetes del Hospital Público Interzonal (Dr. Lucio Molas), se observó que no existían datos acerca del número de pacientes con DG que regresó a control para evaluar su estado metabólico posparto, con evidencia científica que estas mujeres pueden evolucionar a $\mathrm{DM} 2^{2,7}$.

Nos propusimos analizar el porcentaje de mujeres con DG que volvió a control posparto para reclasificar su estado metabólico y determinar cuántas desarrollaron DM2 durante seis años de seguimiento.

\section{MATERIALES Y MÉTODOS}

Estudio descriptivo ambispectivo, realizado desde el $1^{\circ}$ de enero de 2013 al 31 de diciembre de 2019. Se incluyeron mujeres con DG diagnosticadas en el período del $1^{\circ}$ de enero al 31 de diciembre de 2013 en un Hospital Público Interzonal (Dr. Lucio Molas, Santa Rosa, La Pampa, Argentina). Se emplearon como fuentes secundarias el sistema informático de salud de la provincia de La Pampa (SIS) a partir del código O24-4 del CIE-10, partograma e historias clínicas no informatizadas. En las pacientes se investigaron antecedente previo de DG y antecedentes de diabetes en familiares de primer grado (AFD), macrosomía fetal y número de cesáreas.

El diagnóstico de DG se efectuó con dos glucemias en ayunas igual o mayor a $100 \mathrm{mg} / \mathrm{dl}$, o con PTOG alterada'. Se investigó en historia clínica si las embarazadas contaban con medición de $\mathrm{HbA} 1 \mathrm{c}$ alterada en algún momento del embarazo (igual o mayor a 5,6\%) evaluada en el Laboratorio Central del Hospital con normas recomendadas por la Asociación Americana de Diabetes (ADA) ${ }^{5}$.

Al momento en que se diagnosticó la DG (año 2013), se determinaron la edad cronológica, semana de gestación (SG) en la cual se realizó el diagnóstico, presión arterial sistólica (PAS) y diastólica (PAD); también se estableció el índice de masa corporal (IMC: peso/talla²) el cual se clasificó de acuerdo a la semana de gestación según Calvo ${ }^{12}$ en bajo peso, normal, sobrepeso y obesidad. Se evaluó la vía del parto (vaginal o cesárea) y el peso del recién nacido (menor a $2.500 \mathrm{~g}, 2.500-3.999 \mathrm{~g}$ y mayor o igual a $4.000 \mathrm{~g}$ ).

Se excluyeron aquellas pacientes con diagnóstico previo de DM1 o DM2. Se investigó si las pacien- tes con DG diagnosticadas en el año 2013 presentaron hasta el 31 de diciembre de 2019 (seis años) alguna alteración metabólica como DG en nuevo embarazo, glucemia alterada en ayunas (GAA), tolerancia a la glucosa alterada (TGA) o DM2 ${ }^{1}$.

Se efectuaron comparaciones de diversas variables que se obtuvieron en el año 2013 durante el embarazo; posteriormente las pacientes se clasificaron en grupos con control y sin control posparto. Se definió como grupo control posparto aquellas que regresaron a la consulta clínica dentro de los seis años de estudio, y grupo sin control posparto a las que presentaron DG en el año 2013 y no regresaron a efectuar consulta clínica y/o bioquímica verificada en historia clínica o sistema informático hospitalario.

\section{Análisis estadístico}

Se realizó análisis estadístico y se obtuvieron medidas de tendencia central y de dispersión para las variables estudiadas. Para la fase comparativa, se utilizaron las pruebas de Chi-cuadrado, prueba t de Student para comparar promedios y F de Snedecor para comparar varianzas. Se trabajó con un nivel de seguridad del 95\%.

\section{Consideraciones éticas}

El protocolo de estudio lo analizó y aprobó el Comité de Ética e Investigación (CEI) del Departamento de Docencia e Investigación de Hospital Dr. Lucio Molas (Santa Rosa, La Pampa).

\section{RESULTADOS \\ Antecedentes encontrados en las pacientes con DG en el año 2013}

Se identificaron 44 pacientes con DG diagnosticadas en el año 2013 y se investigaron los antecedentes bioquímicos que tenían en su historia clínica o en el SIS hasta el 31 de diciembre de 2019 (seis años).

En la Tabla 1 se observan diversos aspectos clínicos y bioquímicos que tenían las pacientes evaluadas durante el embarazo en el año 2013, sin encontrar diferencia entre grupos en la edad cronológica ni en la semana de gestación (SG) en que se diagnosticó la DG.

Durante el año 2013 hubo 1.238 partos con niños nacidos vivos, lo que representaría el 3,7\% de embarazadas con DG.

En el Gráfico 1 se detalla que, del total de pacientes con diagnóstico de DG, el 54,5\% concurrió 
a control obstétrico o clínico posparto entre seis meses y seis años del seguimiento efectuado.

En el año 2013 el diagnóstico de DG se efectuó en el $32 \%$ de las pacientes mediante dos glucemias basales y en el $68 \%$ mediante la PTOG. Al comparar las pacientes que volvieron a control posparto y las sin control posparto, se observa (Tabla 1) que las pacientes con control tuvieron, cuando se diagnosticaron, medianas más elevadas de glucemia basal (t:10,59; $p<0,001)$, con varianzas distintas y promedios distintos en el test unilateral. No se encontró diferencia entre grupos en la glucemia medida a los 120 minutos en la PTOG (t:1,027; NS). Al 91\% ( $n=42)$ del total de pacientes, en algún momento del embarazo se le solicitó HbA1c y se halló que el 33\% tenía un valor mayor o igual a 5,6\%, que es el punto de corte de buen control metabólico ${ }^{5}$.

También en la Tabla 1 se observa la dispersión en el peso e IMC en los dos grupos al inicio del embarazo, por lo cual se tuvieron en cuenta para la comparación las medianas. El IMC ajustado a la semana de gestación indicó que el 59\% del total de las 44 pacientes tenía sobrepeso u obesidad, siendo más elevado el IMC y la frecuencia de obesidad en el grupo de pacientes con control posparto en el lapso de seis años $(r: 2,103 ; p<0,02)$. Las pacientes del grupo con control posparto al inicio del embarazo tenían valores más elevados de tensión arterial sistólica $(r: 2,629 ; p<0,01)$ y diastólica $(r: 1,965 ; p<0,05)$.

Se distingue en la Tabla 2 que, en el año 2013, el $43,2 \%$ de las pacientes tenía antecedente familiar de diabetes (AFD) y $17 \%$ de DG en embarazo previo, correspondiendo el mayor número de antecedentes a las pacientes del grupo con control posparto. En ambos grupos hubo un alto número de pacientes con cesárea. En el grupo con control posparto hubo mayor número de niños con alto peso para la edad gestacional, al igual que el antecedente de un parto gemelar. De las cuatro madres del grupo con control que tuvieron recién nacidos con alto peso para la edad gestacional, en los seis años de seguimiento, una evolucionó a DM2 y tres presentaron glucosa alterada en ayunas.
En la Tabla 3 se presenta el segundo objetivo, que era determinar cuántas pacientes evolucionaron a DM2; de las 24 pacientes que se investigaron (grupo con control posparto), el 66,7\% presentó durante los seis años de seguimiento alguna alteración metabólica y se destacó la DM2 con 33,3\%. Ilustramos como ejemplo de historia metabólica a dos de las 16 pacientes mencionadas que evolucionaron a DM2. En una de ellas se observó que por tres años (2014 a 2016 inclusive) tuvo glucemias entre 110 y 125 mg/dl compatible con GAA, mientras que la segunda paciente registró durante dos años (2014 y 2015) GAA, en 2016 reiteró DG y en 2017 se manifestó la DM2. Al analizar durante seis años el tiempo que tardaron para volver a control posparto y se reevaluó el estado metabólico, se encontró que sólo dos de las 24 pacientes concurrieron a control a los seis y 12 meses, determinando que la mayoría (nueve de 16) lo hizo a partir de los tres años de haber tenido DG.

En la Tabla 4 se detallan la historia metabólica en las 16 pacientes del grupo con control posparto y el trastorno metabólico que se manifestó durante los seis años que fueron investigadas. Excepto una de las ocho pacientes que se le diagnosticó DM2 al año de control posparto, las demás fueron a partir de los tres años de control al igual que la reiteración de cuadro de DG.

De las ocho pacientes que evolucionaron a DM2 durante el embarazo una tenía peso normal, dos sobrepeso y cinco obesidad ${ }^{12}$. Al momento del diagnóstico de la DM2, sólo una paciente tenía peso normal, una bajo peso y seis habían continuado con sobrepeso/obesidad, a lo cual se asoció hipertensión arterial y/o esteatosis hepática.

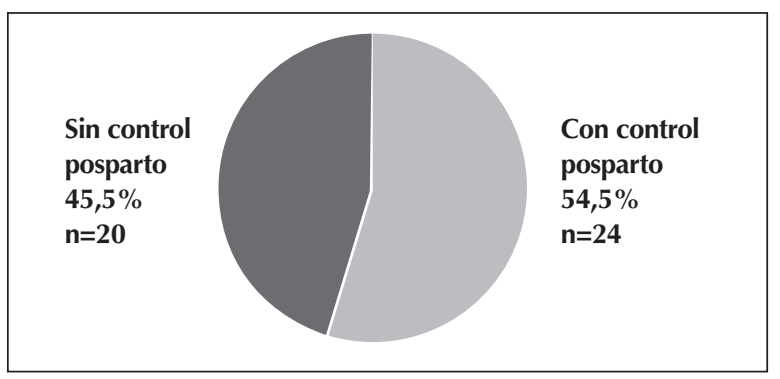

Gráfico 1: Pacientes con diabetes gestacional que concurrieron al control posparto durante seis años de seguimiento $(n=44)$. 


\begin{tabular}{|l|c|c|c|c|c|c|c|c|c|c|}
\hline & \multicolumn{4}{|c|}{ Grupo sin control (n=20) } & \multicolumn{3}{c|}{ Grupo con control (n=24) } & \multicolumn{1}{l|}{} \\
\hline \multicolumn{1}{|c|}{ Variable } & Rango & Media & DS & Md & Rango & Media & DS & Md & t & p \\
\hline Edad (años) & $18-43$ & 29,65 & 7,57 & 28 & $18-44$ & 27 & 7,5 & 27 & 1,162 & NS \\
\hline SG diagnóstico & $20-36,4$ & 28,23 & 3,91 & 28,5 & $20-36$ & 28,92 & 4,86 & 29,1 & 0,69 & NS \\
\hline Peso $(\mathrm{kg})$ & $42,5-124$ & 77,74 & 17,27 & 76,5 & $45-142$ & 90,67 & 20,1 & 86,2 & 3,15 & 0.01 \\
\hline Talla $(\mathrm{m})$ & $1,49-1,69$ & 1,61 & 0,05 & 162 & 1,55 & 1,63 & 0,05 & 1,63 & 1,96 & 0,05 \\
\hline IMC & $16,7-43,4$ & 29,7 & 6,4 & 29,4 & 1,74 & 33,9 & 6,78 & 33,1 & 2,103 & 0,02 \\
\hline PAS $(\mathrm{mmHg})$ & $70-130$ & 103 & 16,25 & & $80-140$ & 115,4 & 15,04 & 115 & 2,629 & 0,01 \\
\hline PAD (mmHg) & 80 & 61,5 & 11,36 & & $50-95$ & 68,47 & 12 & 70 & 1,965 & 0,05 \\
\hline Glucemia mg/dl & $73-168$ & 99,95 & 22,65 & 94 & $79-168$ & 160,10 & 12,62 & 103 & 10,59 & 0,001 \\
\hline PTOG mg/dl & $142-192$ & 160,10 & 12,62 & 159,5 & 145,20 & 165,14 & 18,4 & 162 & 1,027 & $\mathrm{NS}$ \\
\hline HbA1c & $4,8-6$ & 4,21 & 2,19 & 5 & $4-7$ & 4,15 & 2,38 & 5 & 0,12 & $\mathrm{NS}$ \\
\hline SG parto & $37-40$ & 38,5 & & 39,5 & $36-40,2$ & 38,71 & 1,71 & 39 & 0,60 & $\mathrm{NS}$ \\
\hline
\end{tabular}

SG: semana de gestación; IMC: índice de masa corporal; PAS: presión arterial sistólica; PAD: presión arterial diastólica; PTOG: prueba de tolerancia oral a la glucosa.

Tabla 1: Presentación de las pacientes con diabetes gestacional evaluadas año 2013 ( $n=44)$.

\begin{tabular}{|l|c|c|c|c|c|}
\hline \multicolumn{1}{|c|}{ Variable } & \multicolumn{2}{|c|}{$\begin{array}{c}\text { Grupo sin control } \\
(\mathbf{n = 2 0})\end{array}$} & \multicolumn{2}{c|}{$\begin{array}{c}\text { Grupo con control } \\
(\mathbf{n = 2 4 )}\end{array}$} & Total \\
\hline AFD (cantidad) & 8 & $40 \%$ & 11 & $46 \%$ & 19 \\
\hline Antecedente DG (cantidad) & 1 & $5 \%$ & 4 & $17 \%$ & 5 \\
\hline Bajo peso (cantidad) & 1 & $10 \%$ & 1 & 4,1 & 2 \\
\hline Peso normal (cantidad) & 9 & $45 \%$ & 7 & 29,16 & 16 \\
\hline Sobrepeso (cantidad) & 8 & $40 \%$ & 9 & 37,5 & 17 \\
\hline Obesidad (cantidad) & 2 & $10 \%$ & 7 & 29,16 & 9 \\
\hline Macrosomía (cantidad) & 1 & $5 \%$ & 4 & $16,7 \%$ & 5 \\
\hline Cesárea (cantidad) & 14 & $70 \%$ & 16 & $67 \%$ & 30 \\
\hline
\end{tabular}

AFD: antecedentes familiares de diabetes; DG: diabetes gestacional.

Tabla 2: Factores de riesgo presentes en las embarazadas.

\begin{tabular}{|l|c|c|}
\hline \multicolumn{1}{|c|}{ Estado metabólico } & $\mathbf{n}$ & $\mathbf{\%}$ \\
\hline Tolerancia normal a la glucosa & 8 & 33,3 \\
\hline Glucosa alterada en ayunas & 4 & 16,6 \\
\hline Tolerancia a la glucosa alterada & 1 & 4,1 \\
\hline Diabetes gestacional & 3 & 12,5 \\
\hline Diabetes mellitus 2 & 8 & 33,3 \\
\hline Total de pacientes seguidas & 24 & 100 \\
\hline
\end{tabular}

Tabla 3: Evolución de los estados metabólicos en 24 embarazadas con diabetes gestacional evaluadas durante seis años (2013-2019).

\begin{tabular}{|l|c|c|c|c|c|c|c|}
\hline \multicolumn{1}{|c|}{ Evolución metabólica } & $\mathbf{2 0 1 4}$ & $\mathbf{2 0 1 5}$ & $\mathbf{2 0 1 6}$ & $\mathbf{2 0 1 7}$ & $\mathbf{2 0 1 8}$ & $\mathbf{2 0 1 9}$ & Total \\
\hline Glucosa alterada en ayunas (cantidad) & 1 & - & - & 1 & 1 & 1 & 4 \\
\hline Tolerancia a la glucosa alterada (cantidad) & - & 1 & - & - & - & - & 1 \\
\hline Diabetes gestacional (cantidad) & - & - & 3 & - & - & - & 3 \\
\hline Evolucionan a diabetes tipo 2 (cantidad) & 1 & - & 1 & 4 & 2 & - & 8 \\
\hline Subtotales & 2 & 1 & 4 & 5 & 3 & 1 & 16 \\
\hline
\end{tabular}

Tabla 4: Tipo de trastorno metabólico detectado en 16 pacientes con diabetes gestacional de acuerdo al tiempo de evolución de control posparto. 


\section{DISCUSIÓN}

Se investigó la evolución metabólica de 44 mujeres con diagnóstico de DG en un Hospital Público donde se realizaron 1.238 partos con niños nacidos vivos, lo que representó una prevalencia de 3,7\% que se correspondió con similares resultados encontrados en Argentina ${ }^{6}$ y menor al 5 al $18 \%$ en otros países ${ }^{4,13}$.

El $54,5 \%$ de las pacientes con DG diagnosticadas en el año 2013 concurrió tardíamente a efectuar control posparto obstétrico o clínico coincidentes con otros autores ${ }^{4,6}$. Estos resultados evidencian el fracaso comunicacional educativo que se realiza para que vuelvan a control posparto. Consideramos, al igual que otros autores, que deben buscarse otras alternativas sociales y comunicacionales en el sistema de salud para determinar el seguimiento posparto de estas pacientes considerando el riesgo que tiene de evolución a $\mathrm{DM}^{3,8}$

Se encontró, al igual que lo planteado por otros autores ${ }^{4,6}$, que las pacientes del grupo con control posparto seguidas tenían al inicio del embarazo una elevada prevalencia de factores de riesgo para DG como AFD, sobrepeso y obesidad. Una de las falencias de este estudio es que no tenemos evidencias si estas embarazadas habían iniciado el embarazo con exceso de peso, existiendo antecedentes que se desconoce el peso previo a la gestación en el 70\% de las embarazadas que consulta tardíamente ${ }^{12}$.

El diagnóstico de DG se efectuó en el $68 \%$ de las pacientes a partir de la PTOG porque se propuso como el método diagnóstico de elección para la valoración de DG ${ }^{14}$. La HbA1c se solicitó a un elevado número de pacientes en el transcurso del embarazo, y se detectó en el $30 \%$ un valor mayor o igual a $5,6 \%$ para seguimiento 5

\section{Factores de riesgo para DM2 y evolución metabólica detectada en el posparto}

Encontramos, al igual que otros investigadores $^{14}$, que las pacientes con DG estudiadas tenían al inicio del embarazo mayor frecuencia de obesidad y AFD, dos factores de riesgo que se relacionan directamente con la evolución a DM2 ${ }^{14}$, a los cuales se suman la etnia, edad avanzada, factores de riesgo obstétricos como haber padecido DG en embarazo previo ${ }^{14}$, HbA1c elevada y uso de insulina en embarazos previos ${ }^{15}$.

En el año 2013, las medianas de glucemias basales al inicio del embarazo eran mayores en las pacientes del grupo con control posparto lo cual, suma- do a la alta frecuencia de sobrepeso y obesidad de estas pacientes serían dos factores de riesgo para la aparición de DG; a éstos Gorbán de Lapertosa et al. ${ }^{10}$ asocian la hipertrigliceridemia que no fue objeto de estudio en este trabajo. Retnakarán et al. ${ }^{16}$ estudiaron 487 mujeres embarazadas que acudieron preparto a efectuar screening para DG mediante una PTOG. Cuando las controlaron nuevamente a los tres meses posparto, determinaron que la prevalencia de intolerancia a la glucosa o DM2 fue proporcional al estado metabólico que tenían en el preparto. Kugishima et al. ${ }^{8}$ plantearon una controversia en el diagnóstico de seguimiento posparto en el empleo de $\mathrm{HbA} 1 \mathrm{c}$ y PTOG, y concluyeron que son variables independientes y que la PTOG aún posee mayor sensibilidad. Se observó que las pacientes del grupo con control posparto al inicio del embarazo también tenían valores más elevados de tensión arterial lo que se sumaría a la presencia de un síndrome metabólico. Estos resultados permiten al equipo de salud realizar educación diabetológica e intervenir para modificar los estilos de vida de estas pacientes por medio de programas diseñados para tal fin. El objetivo es reducir el peso corporal con plan alimentario y actividad física para disminuir la insulinorresistencia y el agotamiento del páncreas ${ }^{6,17,18}$.

En respuesta al segundo objetivo propuesto, se determinó que el 33,3\% de las pacientes con DG del grupo con control posparto evolucionó a DM2, similar a otros estudios ${ }^{4,19}$ y con mayor frecuencia al estudio de Kugishima7, quien con un tiempo de seguimiento similar al realizado en este estudio haIló 10\% de evolución a DM2. Otros autores ${ }^{14}$ determinaron, en pacientes con DG seguidas durante 15 años, que el riesgo de aparición de DM2 llegó al $25,8 \%$ a diferencia de embarazadas sin antecedentes de DG.

Consideramos que el número de pacientes evaluadas es bajo, aunque la fortaleza del estudio es que se las investigó por seis años en el posparto y se determinó que el 66,7\% presentó alguna alteración metabólica.

El control posparto se realizó fuera del tiempo propuesto por el equipo de salud tratante ${ }^{1,5}$, y quedó de manifiesto que en seis de las ocho pacientes que evolucionaron a DM2 se perdió la oportunidad de que modificaran cambios en el estilo de vida porque continuaron con sobrepeso/obesidad y agregaron otros factores de riesgo cardiovascular lo cual, a decir de Sanderson ${ }^{11}$, en estas pacientes se perdió la posibilidad de prevenir la evolución a la DM2. 
Respecto de la evolución metabólica, al igual que otras investigaciones ${ }^{8}$, encontramos que el $12,5 \%$ de estas pacientes presentó nuevamente DG a los tres años del primer diagnóstico de DG. Otro aspecto que rescatamos para conocer la historia metabólica evolutiva de las pacientes es que antes de llegar al diagnóstico de DM2 atravesaron distintos cambios metabólicos como fue la repetición de DG, TAG o GAA. La aparición de DM2 en el grupo con control posparto ocurrió en mayor medida a partir de los tres años que coincide con otra investigación ${ }^{15}$ en la cual se evaluaron a los tres meses, uno y tres años la función de las células beta pancreáticas, sensibilidad a la insulina y glucemia basal. Estos autores ${ }^{15}$ determinaron a los tres años cambios metabólicos que transcurrían desde GAA, TAG, DG hasta la posibilidad de DM2 luego de la gestación observando, con el transcurso del tiempo, el detrimento progresivo de la función de las células beta y la reducción de la sensibilidad a la insulina en cada etapa de progresión de esta alteración metabólica. De esta manera, se favorece la hipótesis que enuncia que la alteración glucémica preparto en las pacientes con DG es mayor que en aquellas a quienes se diagnosticó TAG, y que las pacientes con TAG evolucionan a las $D^{15}$. Queda demostrado que cualquier grado de disglucemia en el embarazo predice un aumento de riesgo futuro de evolución a DM2 en los años posteriores al mismo ${ }^{16,20}$.

\section{Antecedentes obstétricos}

Detectamos que el $17 \%$ de las pacientes tenía antecedente de DG en gestas previas, similar a otra investigación en Argentina ${ }^{6}$. Entre los antecedentes obstétricos, la macrosomía fetal fue del 11,4\%, predominando en pacientes del grupo con control, las que en su totalidad evolucionaron a DM2 o glucosa alterada en ayunas. Similar resultado se determinó recientemente en un estudio con mayor número de pacientes en ciudades de Argentina ${ }^{10}$. Stoggiani ${ }^{9}$ atribuye la macrosomía fetal a la obesidad, aunque otros autores consideran que también debe considerarse la hipertrigliceridemia ${ }^{21}$.

La alta frecuencia de cesáreas realizadas en todas las pacientes estudiadas podría atribuirse a la presencia de sobrepeso u obesidad que padecían y a la macrosomía fetal6,8,9. La DG y la macrosomía fetal podrían prevenirse al controlar tempranamente en el embarazo el peso para prevenir la obesidad y sus factores de riesgo con cambios en el estilo de vida12. En las pacientes con DG tam- bién juega un rol preponderante el control del IMC de acuerdo a la edad gestacional2,6.

Para el clínico, el seguimiento de mujeres con DG tiene un sentido preventivo del posible desarrollo del síndrome metabólico y sus consecuencias a largo plazo si no se actúa prontamente en este proceso evolutivo, pudiendo presentar las pacientes entre una de sus consecuencias 1,5 veces más riesgo cardiovascular que las mujeres con tolerancia a la glucosa normal22,23. En un estudio de 32 años de seguimiento a pacientes con DG, los autores sitúan al médico clínico como conocedor de los antecedentes e interventor en la prevención de la aparición de las complicaciones cardiovasculares ${ }^{13}$. Es posible que determinar factores de riesgo como los AFD e intervenir sobre los mismos pueda disminuir la prevalencia de DM2 ya que serían un indicador del aumento de esa enfermedad en la población ${ }^{24}$.

Consideramos como limitación de este trabajo que el número de pacientes seguido es bajo y que escasean registros de variables clínicas y de laboratorio por la falta de consulta lo que impide universalizar las conclusiones, por lo cual es necesario efectuar nuevas investigaciones.

\section{CONCLUSIONES}

Hasta la actualidad no existía evidencia en la región pampeana del número de pacientes con DG que vuelve al control posparto y cuántas de ellas evolucionan a DM2. El 54,5\% de las pacientes con DG que concurrió a control metabólico posparto presentaba mayor número de factores de riesgo obstétricos y cardiometabólicos, y el $33,3 \%$ evolucionó a DM2 en el transcurso de seis años de seguimiento. Estos resultados demuestran la necesidad de buscar alternativas sociales y comunicacionales para disminuir el número de pacientes que no vuelve a control posparto dado que se encontró algún grado de alteración metabólica, mayormente a partir de los tres años de haber sido diagnosticada la DG.

Consideramos que determinar tempranamente en el posparto algún grado de trastorno metabólico mediante glucemia basal, presencia de sobrepeso/obesidad, valores de presión arterial elevada y antecedentes familiares de diabetes y gineco obstétricos brindaría la posibilidad de diagnosticar, en mujeres con antecedentes de DG, tempranamente la DM2 que pudiera estar en una fase subclínica e intervenir sobre la hiperglucemia 
que no tratada aumenta el riesgo cardiovascular. Queda pendiente efectuar un estudio prospectivo que determine la relación que tiene el antecedente de macrosomía fetal en el desarrollo futuro de algún grado de intolerancia a la glucosa.

\section{BIBLIOGRAFÍA}

1. Guías de diagnóstico y tratamiento de la diabetes gestacional. ALAD 2016. Rev ALAD 2016; 6:155-69.

2. Chávez-Courtois M, Chelsea G I, Romero-Pérez G, et al. Experiencia y percepciones de la diabetes gestacional y su automanejo en un grupo de mujeres multíparas con sobrepeso. Ciência \& Saúde Coletiva 2014; 19(6):1643-1652.

3. Nielsen K, Kapur A, Damm P, et al. From screening postpartum follow-up the determinants and barriers for gestational diabetes mellitus (GDM) services, a systematic review. BMC Pregnancy and Childbirth 2014; 14:41. Disponible en: http://www. biomedcentral.com/1471-2393/14/41.

4. Noctor E, Crowe C, Carmody L, et al. Abnormal glucose tolerance post-gestational diabetes mellitus as defined by the International Association of Diabetes and Pregnancy Study Groups criteria. European Journal of Endocrinology 2016; 175:287-297.

5. American Diabetes Association. Management of diabetes in pregnancy: standards of medical care in diabetes. Diabetes Care 2019; 42 (Suppl 1):S165.

6. Gorbán de Lapertosa S, Alvariñas J, Elgart J, et al. Educación terapéutica de mujeres con diabetes gestacional (Edugest): datos correspondientes al período de reclutamiento. Revista de la Sociedad Argentina de Diabetes 2019; Vol 53, Nº 3 Sup.

7. Metzger BE, Gabbe SG, Persson B, et al. International association of diabetes and pregnancy study groups recommendations on the diagnosis and classification of hyperglycemia in pregnancy. Diabetes Care 2010; 33(3):676-82.

8. Kugishima $Y$, Ichiro $Y$, Hiroshi $Y$, et al. Risk factors associated with the development of postpartum diabetes in Japanese women with gestational diabetes. BMC Pregnancy and Childbirth 2018; 18:19.

9. Stogianni $A$, Lendahls $L$, Landin-Olsson $M$, et al. Obstetric and perinatal outcomes in pregnancies complicated by diabetes, and control pregnancies, in Kronoberg, Sweden. BMC Pregnancy and Childbirth 2019; 19(1):159. Disponible en: https://doi. org/10.1186/s12884-019-2269-8.

10. Gorbán de Lapertosa S, Alvariñas J, Elgart JF, et al. The triad macrosomia, obesity, and hypertriglyceridemia in gestational diabetes. Diabetes Metab Res Rev. 2020 Feb; 18:e03302. DOI: 10.1002/dmrr.3302.

11. Sanderson $\mathrm{H}$, Loveman $\mathrm{E}$, Colquitt $\mathrm{J}$, et al. Improving uptake of postnatal checking of blood glucose in women who had gestational diabetes mellitus in Universal Healthcare Settings: a systematic review. J Clin Med 2019; 8:4. DOI:10.3390/jcm8010004 www.mdpi.com/journal/jcm.
12. Calvo, EB, López LB, Balmaceda $Y$, et al. Reference charts for weight gain and body mass index during pregnancy obtained from a healthy cohort. The Journal of Maternal-Fetal \&Neonatal Medicine 2009; 22(1):36-42.

13. Benhalima K, Lens $\mathrm{K}$, Bosteels J, et al. The risk for glucose intolerance after gestational diabetes mellitus since the introduction of the IADPSG criteria: a systematic review and metaanalysis. J Clin Med 2019; 8(9):1431. DOI: 10.3390 /jcm8091431.

14. Lee AJ, Hiscock RJ, Wein P, et al. Gestational diabetes mellitus: clinical predictors and long-term risk of developing type 2 diabetes. A retrospective cohort study using survival analysis. Diabetes Care 2007; 30:878-883.

15. Kramer C.K, Balakumar S, Anthony JH, et al. Each degree of glucose intolerance in pregnancy predicts distinct trajectories of b-cell function, insulin sensitivity, and glycemia in the first 3 years postpartum. Diabetes Care 2014; 37:3262-3269. DOI: 10.2337/dc14-1529

16. Retnakaran R, Ying Qi, Mathew Sermer, et al. Glucose intolerance in pregnancy and future risk of pre-diabetes or diabetes. Diabetes Care 2008; 31:2026-31.

17. O'Reilly S L, LeonardY, Dasgupta K, et al. Diabetes after pregnancy prevention trials: systematic review for core outcome set development. Matern Child Nutr 2020; e12947. Disponible en: wileyonlinelibrary.com/journal/mcn 1 of $11 \mathrm{https} / / / \mathrm{doi}$ org/10.1111/mcn.12947.

18. Wasalathanthri S. Attenuating type 2 diabetes with postpartum interventions following gestational diabetes mellitus. World $\mathrm{J}$ Diabetes 2015; 6(4): 648-653.

19. Oats JN, Beischer NA. The persistence of abnormal glucose tolerance after delivery. Obstet Gynecol 1990; 75:397-40.

20. Carr DB, Newton KM, Utzschneider KM, et al. Modestly elevated glucose levels during pregnancy are associated with a higher risk of future diabetes among women without gestational diabetes mellitus. Diabetes Care 2008; 31:1037-39.

21. Olmos PR, Rigotti A, Busso D, Berkowitz L, Santos JL, Borzone $\mathrm{GR}$, et al. Maternal hypertriglyceridemia: a link between maternal overweight-obesity and macrosomia in gestational diabetes. Obesity (Silver Spring) 2014; 22:2156-63.

22. Sesmilo G, Meler E, Perea V, et al. Glucemia materna en ayunas y resultados adversos del embarazo en una población mediterránea. Acta Diabetol 2017; 54:293-299. Disponible en: https://doi.org/10.1007/s00592-016-0952-z

23. Bellamy L, Casas JP, Hingorani AD, et al. Type 2 diabetes mellitus after gestational diabetes: a systematic review and metaanalysis. Lancet 2009; 373:1773-1779.

24. Cuarta Encuesta Nacional de Factores de Riesgo. Resultados preliminares. $1^{\circ}$ Ed. Ciudad Autónoma de Buenos Aires: Instituto Nacional de Estadísticas y Censos (INDEC), Secretaría de Gobierno de Salud 2019. ISBN 978-950-896-542-4. 